\title{
Modified Optimum Pricing Algorithm with Delayed CSI feedback for Spectrum sharing in D2D based Vehicular Networks
}

\author{
${ }^{1}$ L. Thulasimani, and ${ }^{2}$ Antinita Shilpha Daly A \\ \{1tm.ece@psgtech.ac.in ${ }^{1}$, antinitashilpha@gmail.com $\left.{ }^{2}\right\}$ \\ ${ }^{1}$ Assistant Professor and ${ }^{2}$ PG Wireless Communication Scholar, ${ }^{1 \& 2}$ Department of ECE, PSG College \\ of Technology, Coimbatore, India
}

\begin{abstract}
The next-generation wireless network has to handle a large amount of data transmission resources which need study on new technologies like machine 2 machine communication, device 2 device communication etc., the integration of social network and device 2 device communication in $5 \mathrm{G}$ and upcoming $6 \mathrm{G}$ networks poses very important challenges in various QoS parameter. This paves way for improvement of spectral and energy efficiency for a vehicular communication network. In this paper, clustering schemes and robust power control schemes are utilized to achieve the improved spectral and energy efficiency. Clustering schemes are established to form stable clusters and pricing-based algorithms are used for achieving robust power control. The two robust power control algorithms the fixed price algorithm and optimized price algorithm are analyzed. And to compromise the trade-off produced by these algorithms, a pricing-based algorithm with delayed channel state information feedback for D2D (Device-to- Device) based vehicular communication network is developed in this paper. The results obtained shows that the proposed algorithm is effective.
\end{abstract}

Keywords: Spectral efficiency, energy efficiency, pricing algorithm, channel state information.

\section{Introduction}

Vehicular communication is a technology using which vehicles can communicate with the moving parts of the traffic around them. The main purpose of V2X (Vehicle-to- Everything) technology is improving road safety, energy saving and traffic efficiency on the roads. While using this technology, the V2X systems can convey important information to the driver like information regarding inclement weather, accidents that has happened in nearby places, road condition in which the vehicle is travelling and various other dangerous activities of the nearby vehicles.

The physical and medium access control (MAC) layers of the vehicular networks were originally made for low mobility networks. This leads to various issues in the network [8], [9]. These issues are addressed by recent projects of 3GPP which uses long-term evolution (LTE) networks for the vehicular communication. The sum capacity of the V2I (Vehicle-toInfrastructure) links and the reliability of the $\mathrm{V} 2 \mathrm{~V}$ (Vehicle- to-Vehicle) links can be maximized by considering the channel state information (CSI) that is fed back periodically 
[6]. To reduce the signalling overhead in the vehicular networks, slow fading CSI based resource allocation scheme has been developed in [5]. The green vehicular network for smart and green cities mainly focuses on the maximization of energy efficiency which is a main performance indicator for the system design [1]. The vehicular communication network which involves the use of both $802.11 \mathrm{p}$ standard for the ad-hoc communication and long-term evolution for the back-end based communication is developed [2], [3]. For vehicular networks, Time division multiple access (TDMA) is the commonly used multiple access technique. The TDMA technology uses flexible time slot scheduling for collision- free resource allocation [4]. A better clustering approach which involves in both the geographical information and the speed is studied in [7], [10].

This paper mainly concentrates on optimizing the spectral and energy efficiencies using the optimum pricing algorithm with delayed CSI feedback.

\section{System Model}

The system model considered for this project is depicted in Fig.1. This system model is a cluster-based hybrid architecture. The Fig.1 represents a unidirectional two-lane road with the base station (BS) located on one side of it. The road section depicted in the figure comes under the communication service range of the BS.

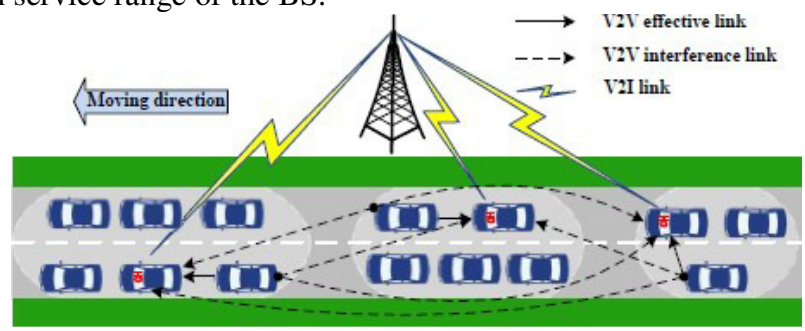

Fig.1 System Model

In this paper, Time Division Multiple Access (TDMA) technique is considered for allocating spectrum resources to various vehicles using flexible time-slot scheduling. Here a round robin time scheduling which is based on node ID is used. The distances between the clusters are based on truncate exponential distribution which excludes values exceeding a certain threshold value. The velocity of the vehicles is based on the constant speed motion model in every time slot.

Each cluster consists of a cluster head $(\mathrm{CH})$ and several cluster members $(\mathrm{CM})$. The vehicular node with the smallest relative mobility will be chosen as the Cluster Head. The nodes adjacent to the $\mathrm{CH}$ which comes within the cluster head effective communication range are said to be its cluster members. The $\mathrm{CMs}$ for a $\mathrm{CH}$ are determined using the

received signal strength (RSS) criterion which combines the velocity and location information. Based on this, the nodes having relatively smaller speed difference with the $\mathrm{CH}$ are chosen to be its CM. The number of CMs per cluster is limited to each cluster to prevent long service waiting in areas with high node density. As the cluster system is based on TDMA technology, a $\mathrm{CH}$ can communicate with a $\mathrm{CM}$ of its own cluster using one time slot. If the $\mathrm{CMs}$ want to communicate with their $\mathrm{CH}$, they can communicate based on the sequentially 
allocated time sequence. All the remaining clusters also use the same time scheduling strategy to communicate within the clusters. The system model is said to be a cluster-based hybrid architecture because the vehicles in the cluster are assumes to use two interfaces for communication. The two interfaces are IEEE 802.11p and LTE. The IEEE 802.11p interface is used by the $\mathrm{CM}$ s to communicate with their $\mathrm{CH}$ and for the $\mathrm{CH}$ to communicate with the CMs. Whereas the LTE interface is used by the CHs to communicate with the BS.

\section{Pricing Based RPC Scheme}

The RPC scheme analyzed in this paper is the pricing-based algorithm which involves imperfect CSI. And the signals transmitted to the receiver from the transmitters are considered to be in line of sight propagation.

\section{A. Fixed Price $C$ algorithm}

The pricing-based scheme involves in introducing a parameter called Price which will be introduced as a penalty to the total power consumption for the sum rate. The clusters interference links are formulated to be probability constraints due to the rapid changes in the mobile channels. The average SINR or the long term SINR parameter is considered instead of the real-time SINR. This is because the real-time SINR is difficult to be obtained for a rapidly changing network. To provide a better balance between the average sum rates and the power consumed, the network price $\mathrm{C}$ has to be greater than or equal to zero. Based on the price $\mathrm{C}$ parameter, the network utility is given as

$$
\max U(p)=\sum_{i=1}^{N} \log 2(1+\mathbb{P}(\mathrm{p}))-\mathrm{C}\left(\sum_{i=1}^{N} \mathrm{ci} * \mathrm{pi}+\mathrm{Pc}\right)(1)
$$

$\mathbb{P}(\mathrm{p})$ is the average SINR or the long term SINR of the effective vehicular links. The amplifier coefficient for the active cluster members is given as ci which as to be greater than 1 (ci>1). The maximum transmitter power and the circuit power consumption for all the active cluster members are given as pi and Pc. For all the cluster heads an interference threshold (Ith) is considered because the above equation involves the interferences in the cluster heads which is caused by the cluster members of the neighbouring clusters.

The fixed price algorithm turns out to be a sum throughput maximization algorithm when the price value tends to zero. When $\mathrm{C}$ tends to zero, the consuming power cost becomes almost zero which makes the algorithm a sum rate maximization work. And when $\mathrm{C}$ tends to infinity, the algorithm changes to energy efficient algorithm. But it does not have a good power allocation at maximum values of $\mathrm{C}$. For channels with severe channel fading, the probability constraint would become nonconvex and intractable. Hence, the Bernstein approximation which is a convex approximation strategy is introduced into this algorithm. Along with the Bernstein approximation, Successive convex approximation is also included to correct the intractable nature of the long-term sum rates. And based on all these constrains the spectral efficiency of the vehicular network is formulated as

$\mathrm{SE}(\mathrm{F})=\mathrm{B} *(\log (\mathrm{C}+\mathrm{SINR}(\mathrm{k})) / \log (2) \mathrm{C})$

And using (2) the energy efficiency is formulated as given below.

$\mathrm{EE}(\mathrm{F})=\mathrm{SE}(\mathrm{F}) /\left(\mathrm{ci}{ }^{*} \exp (\mathrm{Temp}(\mathrm{F})+\mathrm{PC})\right)(3)$

Where the range of $\mathrm{F}$ varies from zero to ten. The optimal power storage area for each iteration is defined as Temp.

B. Optimum Price C algorithm 
In the fixed price $\mathrm{C}$ algorithm, the maximum energy efficiency was obtained only when $\mathrm{C}$ tends to maximum. In the optimum price $\mathrm{C}$ algorithm, the energy efficiency maximization is obtained directly by adding an extra step which involves in calculating an optimum value for the parameter price $\mathrm{C}$. The nonlinear and intractable nature remains in the power vector even after the optimization by the fixed price $\mathrm{C}$ algorithm. This is solved by considering logarithmic conversion of the power vector elements. This conversion can change the problem into a standard concave maximization strategy. The log transformation is considered to be a distributed power iteration.

The major issue in the previous algorithm was the trade-off between the spectral and energy efficiencies which occurs as the value of $\mathrm{C}$ increases. To overcome this issue, the optimal price $\mathrm{C}$ algorithm follows an iterative process to calculate an optimal value for the price parameter $\mathrm{C}$ for every iteration. The optimum price $\mathrm{C}$ parameter for the kth iteration utility function is given as

$$
\begin{array}{cl}
\mathrm{C}(\mathrm{k}+1)=\mathrm{Rs}(\mathrm{ep}(\mathrm{k})) / \quad \mathrm{ci} * \mathrm{ep}(\mathrm{k})+\mathrm{Pc} & \text { (4) } \mathrm{Rs}(\mathrm{ep}) \text { which defines the achievable } \\
\text { long-term sum rate is given as } &
\end{array}
$$

$\operatorname{Rs}(\mathrm{ep})=\log 2(1+\not{\gamma}(\mathrm{p})) \quad$ (5) First the counter value $\mathrm{k}$ is initialized as $\mathrm{k}=1$ and for each iteration it is updated as $k=k+1$. And the parameter price $C$ is initialized as $C(1)=0.1$ and it is updated for each iteration using the equation (4). Using equations (2) and (3), the efficiencies are determined.

\section{Optimum Price Algorithm With Delayed CSI Feedback}

This section describes the proposed optimum price algorithm with delayed CSI feedback. The channel state information (CSI) is an important criterion that defines the parameters of the channels of a particular communication link. For resource allocation design CSI is very a major parameter required at the base station. In vehicular networks due to the high vehicle mobility, it is difficult to obtain the accurate CSI. The previously analyzed fixed price $\mathrm{C}$ and optimum price $\mathrm{C}$ algorithms are based on imperfect CSI. The imperfect CSI turns out to be major problem when it comes to practical applications. It mainly affects the interference alignment of the communication system. When the imperfect CSI is used in the analysis of the communication network it causes unignorable errors in the vehicular networks.

To overcome these issues, the optimum price algorithm with delayed CSI feedback is proposed in this paper. In the optimum price algorithm with delayed CSI feedback, the channel state information of the vehicular links is reported to the base station periodically. For the links formed in between the vehicles and the base station i.e., for the Vehicle-toinfrastructure (V2I) links the channel state information can be estimated by the base station. This is because for the V2I links, the links are formed directly between the base station and the vehicle. Hence for the V2I links the CSI feedback is not required. For the links between formed in between the vehicles i.e., the V2V links the channel state information of these links are reported to the base station with a feedback period. Since this is a cluster-based architecture involving $\mathrm{CH}$ and $\mathrm{CMs}$, the CSI of the cluster members are reported through the cluster heads to the base station.

A feedback period of $\mathrm{T}=1 \mathrm{~ms}$ is initialized first. The CSI feedback part which is the fast fading model over the feedback period $\mathrm{T}$ is given as 
$h 1=\epsilon h+e \quad(6)$

where $\mathrm{h} 1$ represents the current channel fast fading and $\mathrm{h}$ represents the previous channel fast fading. e represents the distributed channel correlation and $\epsilon$ quantifies the channel correlation between the two consecutive time slots. The zero- order bessel function for the channel fast fading is determined using the below given formula

$\mathrm{j} 1=2 * \mathrm{pi} * \mathrm{fc} * \mathrm{~T} / \mathrm{c} * \mathrm{v} \quad$ (7) The value of bessel function is formulated as

$\epsilon=J 0(2 \pi f d T)$

Based on these above equations the basic parameters for the delayed CSI feedback is evaluated. After this, the algorithm follows the optimum price algorithm. Using equations (4) and

(5) the optimum value for the parameter price $C$ is calculated. And using the equations (2) and (3) the efficiencies are calculated and analyzed.

\section{Simulation Results}

This section presents the simulation results obtained for the fixed price $\mathrm{C}$, optimum price $\mathrm{C}$ and the optimum price with delayed CSI feedback. The simulation results are obtained using MATLAB software. The cluster-based system model is considered with five clusters. The major parameters for the simulation are listed in Table 1.1.

Table 1.1 Simulation Parameters

\begin{tabular}{|l|l|}
\hline Parameters & Value \\
\hline Carrier frequency & $5.9 \mathrm{GHz}$ \\
\hline Bandwidth & $1 \mathrm{GHz}$ \\
\hline Radio range & $300 \mathrm{~m}$ \\
\hline CSI feedback period & $1 \mathrm{~ms}$ \\
\hline Average vehicle speed & $25 \mathrm{~m} / \mathrm{s}$ \\
\hline Mean of background noise & $-30 \mathrm{dBm}$ \\
\hline Maximum CM transmitter power & $0.05 \mathrm{~W}$ \\
\hline $\begin{array}{l}\text { Log-normal shadowing standard } \\
\text { deviation }\end{array}$ & $10 \mathrm{~dB}$ \\
\hline Pathloss exponent & 3 \\
\hline
\end{tabular}




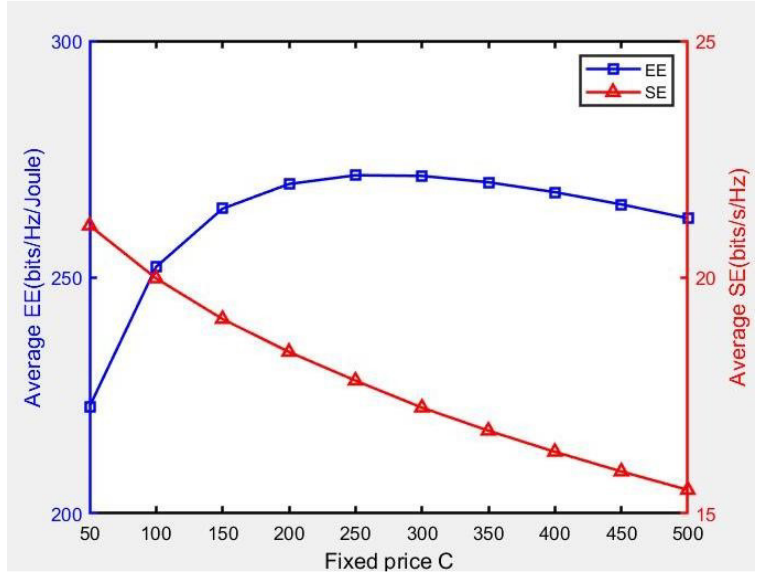

Fig.2 Average EE and SE for fixed prices $C$

The Fig. 2 represents the average energy and spectral efficiencies obtained for the fixed price $\mathrm{C}$ algorithm. The efficiency is compared with different price values. From the above figure it is observed that the fixed price $\mathrm{C}$ algorithm produces a trade-off between the spectral and energy efficiencies. As the value of $\mathrm{C}$ increases, the energy efficiency increases but the spectral efficiency decreases. A maximum spectral efficiency is obtained only when $\mathrm{C}$ tends to zero. The average energy and spectral efficiencies for optimum price $\mathrm{C}$ algorithm is depicted in Fig.3. From this figure it can be observed that the energy efficiency increases monotonically as the iterations increases. But the average spectral efficiency is very minimum when compared with the energy efficiency and it remains unchanged for every iteration. Since the pricing-based algorithm is a robust power control scheme, it reduces the power level relatively which in turn improves the background noise in the SINR expression. This finally leads to smaller sum rates.

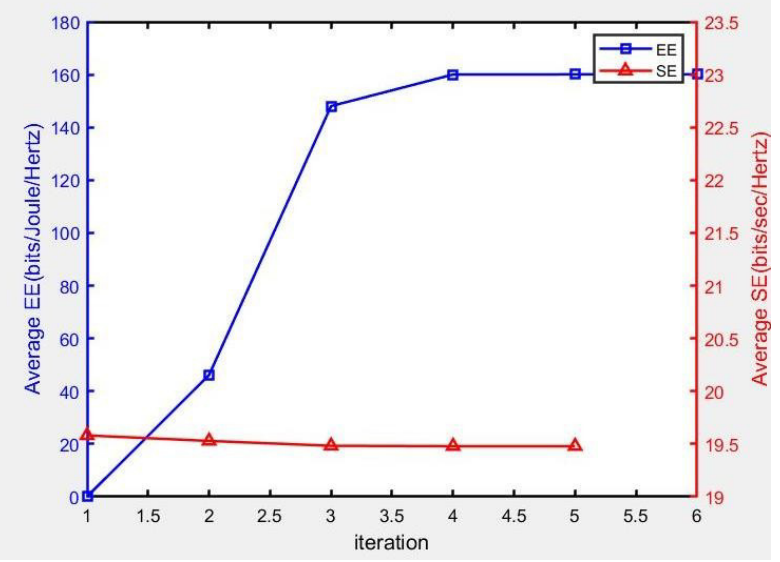

Fig.3 Average EE and SE for optimum price $\mathrm{C}$ algorithm

The Fig.4 shows the average efficiencies obtained for the proposed optimum price C algorithm with delayed CSI feedback. The analyses of the obtained results show that the 
proposed work provides a good balance between the spectral and energy efficiencies. Both the efficiencies work together but as the iterations increase it follows a downward trend.

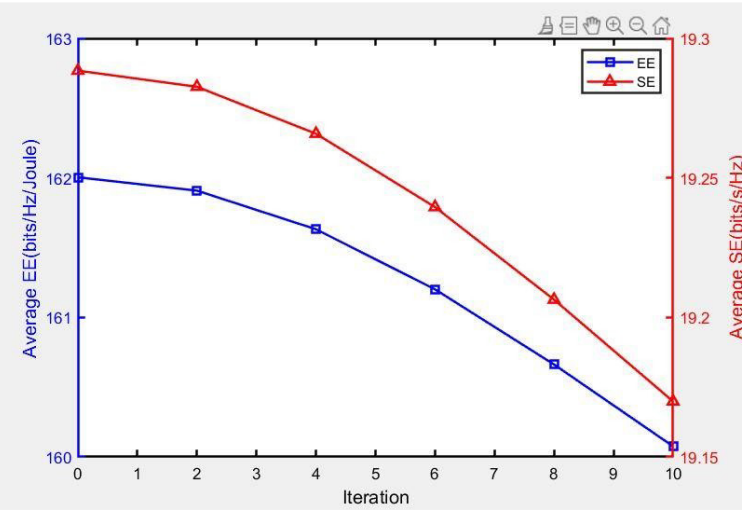

Fig.4 Average EE and SE for optimum price C with delayed CSI feedback algorithm

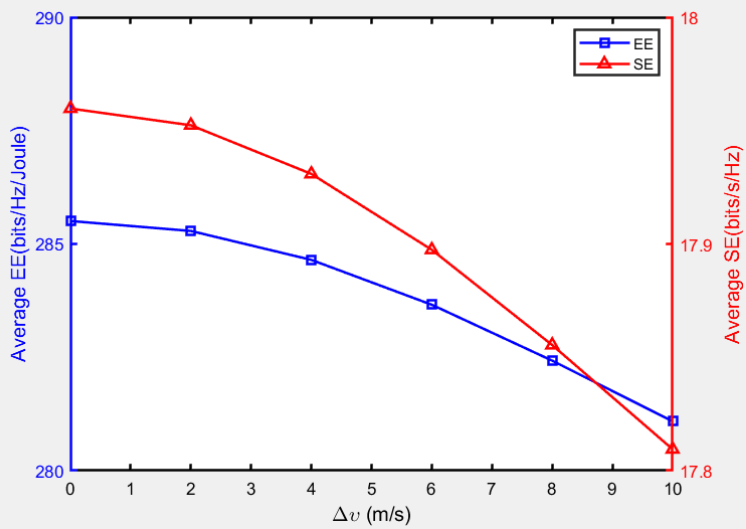

Fig.5 Average EE and SE Vs Relative speed 


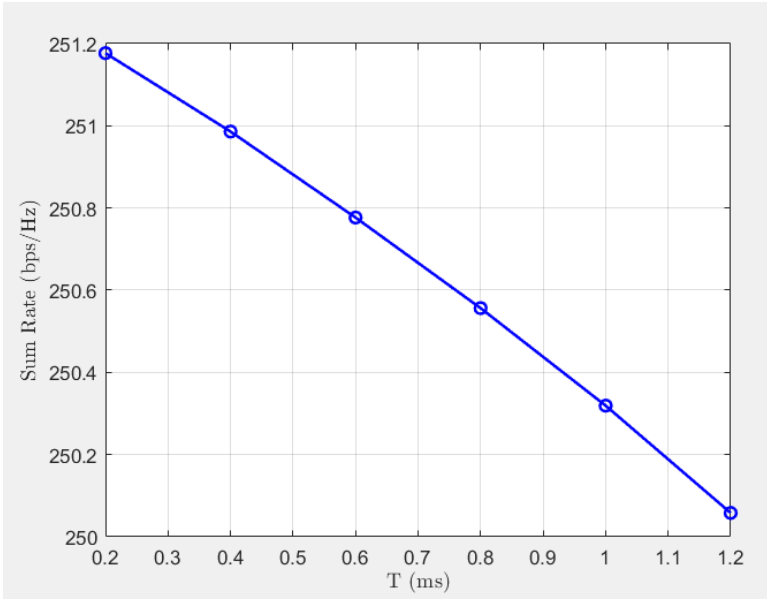

Fig.6 Sum rate of vehicular links Vs Sampling Time

The average spectral and energy efficiencies are compared with the relative speed of the vehicles in Fig.5. The relative speed is calculated in between the cluster heads and the cluster members. The speed of the cluster heads is considered as $20 \mathrm{~m} / \mathrm{s}$ and the speed of the cluster members varies from $20 \mathrm{~m} / \mathrm{s}-30 \mathrm{~m} / \mathrm{s}$. From the Fig.5, we observe that the efficiencies decrease as the relative motion between the $\mathrm{CH}$ and $\mathrm{CM}$ increases. This shows the Doppler effect occurring in the vehicular network due to the relative motion.

The Fig.6 represents the sum rate of the vehicular links for different feedback time (T). The increasing feedback time reduces the sum rate of the vehicular links due to delay occurring in sending the CSI feedback. From observing all the results obtained, it is clear that the proposed optimum price algorithm with delayed CSI feedback provides a good balance between the system efficiencies.

\section{Conclusion}

The joint clustering and robust power control scheme for D2D based vehicular networks has been analyzed and a RPC scheme involving delayed CSI feedback is proposed in this paper. The algorithms are implemented using MATLAB software. The algorithms for RPC scheme that is fixed price $\mathrm{C}$ and optimum price $\mathrm{C}$ algorithms involving the use of imperfect CSI are analyzed. To overcome the tradeoff caused due to these algorithms, an optimum price $\mathrm{C}$ algorithm with delayed CSI feedback is proposed. By analyzing the results obtained, it is clear that the proposed algorithm provides a good balance between the spectral and energy efficiency. And the proposed algorithm works well for smaller relative speed and with smaller feedback period. For future work, deep neural network can be used to compress CSI and allocate resources. 


\section{References}

[1] Yuan-ai Xie, Zhixin Liu, Kit Yan Chan. "Energy-Spectral Efficiency Optimization in Vehicular Communications: Joint Clustering and Pricing- based Robust Power Control Approach", IEEE transactions on vehicular communication, 2020.

[2] Z. Liu et al., "Efficient QoS support for robust resource allocation in blockchain-based femtocell networks", IEEE transactions on vehicular communication, 2020.

[3] Z. Zhou, F. Xiong, C. Xu, Y. He, and S. Mumtaz, "Energy-efficient vehicular heterogeneous networks for green cities", IEEE Trans. Ind. Inform., vol. 14, no. 4, 2019.

[4] M. Hadd et al., "TDMA-Based MAC Protocols for Vehicular Ad Hoc Networks: A Survey, Qualitative Analysis, and Open Research Issues", IEEE Communication Surveys Tuts., vol. 17, 2018.

[5] Le Liang, Shijie Xie, Geoffrey Ye Li, Zhi Ding, and Xingxing Yu. "Graph-Based Resource Sharing in Vehicular Communication", IEEE transactions on wireless communications, 2018.

[6] Le Liang, Shijie Xie, Geoffrey Ye Li, and Xingxing Yu." Spectrum and Power Allocation with Delayed CSI Feedback", IEEE transactions on wireless communications, 2018.

[7] M. Ren et al., "A unified framework of clustering approach in vehicular Ad Hoc networks," IEEE Transactions on Intelligent Transportation Systems, vol. 19, no. 5, 2018.

[8] Le Liang, Haixia Peng, Geoffrey $\mathrm{Ye} \mathrm{Li}$, and Xuemin (Sherman) Shen. "Vehicular Communications: A Physical Layer Perspective", IEEE transactions on vehicular communication, Vol.66, Issue.12, 2017.

[9] Le Liang, Haixia Peng, Geoffrey Ye Li, and Xuemin (Sherman) Shen. "Vehicular Communications: A Network Layer Perspective", IEEE transactions on vehicular communication, Vol.66, Issue.12, 2017.

[10] S. Ucar et al., "Multihop-cluster-based IEEE 802.11p and LTE hybrid architecture for VANET safety message dissemination," IEEE Transactions on Vehicular Technology, vol. 65, no. 4, 2017. 